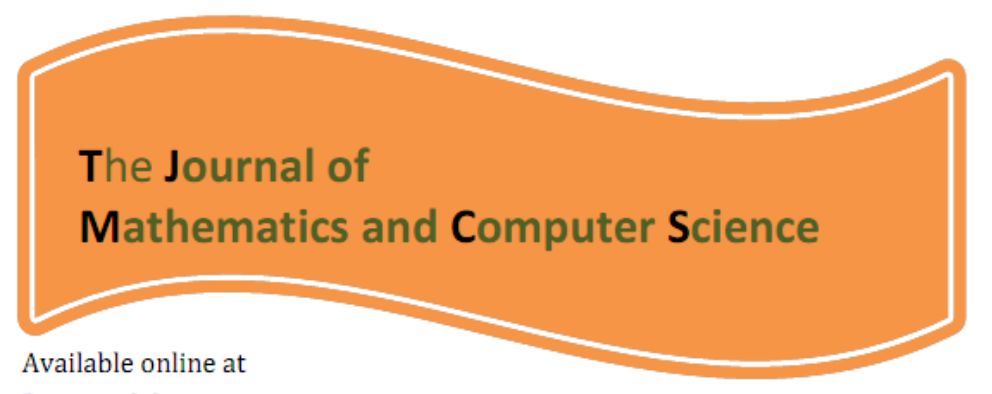

http://www.T]MCS.com

The Journal of Mathematics and Computer Science Vol .3 No.2 (2011) 262 - 277

\title{
The Nehari manifold for a quasilinear elliptic equation with singular weights and nonlinear boundary conditions
}

S.H. Rasouli ${ }^{a}$, K. Fallah ${ }^{b}$

${ }^{a}$ Department of Mathematics, Faculty of Basic Science,

Babol University of Technology, Babol, Iran

e-mail: s.h.rasouli@nit.ac.ir

${ }^{b}$ Department of Mathematics,

Islamic Azad University Ghaemshahr branch, Iran

e-mail: fallahdarabi@yahoo.com

Received: May 2011, Revised: June 2011

Online Publication: December 2011

\section{Abstract}

Using the technique of Brown and $\mathrm{Wu}$ [11], we present a note on the paper [22] by Wu. Indeed, we extend the multiplicity results for a class of semilinear problems to the quasilinear elliptic problems with singular weights of the form:

$$
\begin{cases}-\operatorname{div}\left(|x|^{-a p}|\nabla u|^{p-2} \nabla u\right)=\lambda|x|^{-(a+1) p+c} f(x)|u|^{q-2} u & x \in \Omega, \\ |\nabla u|^{p-2} \frac{\partial u}{\partial n}=|x|^{-(a+1) p+c} g(x)|u|^{r-2} u & x \in \partial \Omega .\end{cases}
$$

Here $0 \leq a<\frac{N-p}{p}, c$ is a positive parameter, $1<q<p<r<p *\left(p *=\frac{p N}{N-p}\right.$ if $N>p$, $p *=\infty$ if $N \leq p), \Omega \subset \mathbb{R}^{N}$ is a bounded domain with smooth boundary, $\frac{\partial}{\partial n}$ is the outer normal derivative, $\lambda \in \mathbb{R} \backslash\{0\}$, and $f(x), g(x)$ are continuous functions which change sign in $\bar{\Omega}$. 
Keywords: Quasilinear elliptic problem; Singular weights; Nehari manifold; Nonlinear boundary condition.

AMS Subject Classification: 35J50, 35J55, 35J65.

\section{Introduction}

The aim of this paper is to prove some existence and multiplicity results of nontrivial nonnegative solutions to the quasilinear elliptic problems:

$$
\begin{cases}-\operatorname{div}\left(|x|^{-a p}|\nabla u|^{p-2} \nabla u\right)=\lambda|x|^{-(a+1) p+c} f(x)|u|^{q-2} u & x \in \Omega, \\ |\nabla u|^{p-2} \frac{\partial u}{\partial n}=|x|^{-(a+1) p+c} g(x)|u|^{r-2} u & x \in \partial \Omega .\end{cases}
$$

where $0 \leq a<\frac{N-p}{p}, c$ is a positive parameter, $1<q<p<r<p *\left(p *=\frac{p N}{N-p}\right.$ if $N>p$, $p *=\infty$ if $N \leq p), \Omega \subset \mathbb{R}^{N}$ is a bounded domain with smooth boundary, $\frac{\partial}{\partial n}$ is the outer normal derivative, $\lambda \in \mathbb{R} \backslash\{0\}$, and $f(x), g(x)$ are are satisfying the following assumptions:

(H1) $f(x) \in C(\bar{\Omega})$ with $\|f\|_{\infty}=1$;

(H2) $g(x) \in C(\partial \Omega)$ with $\|g\|_{\infty}=1$.

For the regular case, that is, when $a=0$ and $c=p$ this problem arise in some physical models like the flow of non-Newtonian fluids: pseudo-plastic fluids correspond to $p \in(1,2)$ while dilatant fluids correspond to $p>2$. The case $p=2$ expresses Newtonian fluids [5]. On the other hand, quasilinear elliptic problems like (1) appears naturally in several branches of pure and applied mathematics, such as the study of optimal constants for the Sobolev trace embedding (see $[13,17,18,19]$ ); the theory of quasiregular and quasiconformal mappings in Riemannian manifolds with boundary (see [16, 20]); non-Newtonian fluids, reaction diffusion problems, flow through porus media, nonlinear elasticity, glaciology, etc. (see $[4,5,6,14]$ ).

The motivation for our investigation is the case $a=0$ and $c=p=2$ ( linear operator) that was studied by $\mathrm{Wu}[22]$. In the recent paper, Brown and $\mathrm{Wu}$ [11] studied the multiplicity results of nontrivial nonnegative solutions for a semilinear elliptic system. Here we focus on further extending the study in [11] for the quasilinear elliptic problem involving the singular weights. Due to this singularity in the weights, the extensions are challenging and nontrivial. Indeed, motivated by [22], and using recent ideas from [11], we shall stablish the existence and multiplicity results for problem (1). In the case when $a=0$ and $c=p=2$ similar 
problems (with Dirichlet or Neuman boundary condition ) have been studied by Drabek et al. [7,8], Ambrosetti-Brezis-Cerami [2] using variational methods and by Amman and Lopez-Gomez [3] by using global bifurcation theory.

In recent years, several authors use the Nehari manifold to solve semilinear and quasilinear problems ( see $[1,9,10,11,21,21,23,24]$ ). Brown and Zhang [12] have studied a subcritical semilinear elliptic equation with a sign-changing weight function and a bifurcation real parameter in the case $a=0$ and $c=p=2$ and Dirichlet boundary conditions. Exploiting the relationship between the Nehari manifold and fibering maps (i.e., maps of the form $t \longmapsto J_{\lambda}(t u)$ where $J_{\lambda}$ is the Euler function associated with the equation), they gave an interesting explanation of the well-known bifurcation result. In fact, the nature of the Nehari manifold changes as the parameter $\lambda$ crosses the bifurcation value. In this work, we give a variational method which is similar to the fibering method ( see $[15]$ or $[9,12]$ ) to prove the existence of at least two nontrivial nonnegative solutions of problem (1). In particular, by using the method of [11], we do this without the extraction of the Plais-Smale sequences in the Nehari manifold as in $[1,22]$.

This paper is divided into three sections, organized as follows. In section 2, we give some notation, preliminaries, properties of the Nehari manifold and set up the variational framework of the problem. In section 3, we give our main result.

\section{Variational setting}

Now, we are setting some spaces and their norms. If $\alpha \in \mathbb{R}$ and $l \geq 1$, we define $L^{l}\left(\Omega,|x|^{\alpha}\right)$ as being the subspace of $L^{l}(\Omega)$, of the Lebesgue measurable functions $u: \Omega \rightarrow \mathbb{R}$, satisfying

$$
\|u\|_{L^{l}\left(\Omega,|x|^{\alpha}\right)}=\left(\int_{\Omega}|x|^{\alpha}|u|^{l}\right)^{\frac{1}{l}}<\infty .
$$

If $1, p<N$ and $-\infty<a<\frac{N-p}{p}$, we define $W=W_{0}^{1, p}\left(\Omega,|x|^{-a p}\right)$, the completion of $C_{0}^{\infty}(\Omega)$, with respect to the norm

$$
\|u\|=\left(\int_{\Omega}|x|^{-a p}|\nabla u|^{p} d x\right)^{\frac{1}{p}} .
$$

Throughout this paper, we set $\mathrm{C}$ and $\bar{C}$ be the best Sobolev and the best Sobolev trace constants for the embedding of $W_{0}^{1, p}\left(\Omega,|x|^{-a p}\right)$ in $L^{q}\left(\Omega,|x|^{-a p}\right)$ and $W_{0}^{1, p}\left(\Omega,|x|^{-a p}\right)$ 
in $L^{r}\left(\partial \Omega,|x|^{-a p}\right)$, respectively. First we give the definition of the weak solution of (1).

Definition 2.1. We say that $u \in W$ is a weak solution to (1) if for any $v \in W$ with $v \geq 0$ we have

$$
\begin{aligned}
\int_{\Omega}\left(|\nabla u|^{p-2}|x|^{-a p} \nabla u . \nabla v\right) d x & =\lambda \int_{\Omega}|x|^{-(a+1) p+c} f(x)|u|^{q-2} u v d x \\
& +\int_{\partial \Omega}|x|^{-(a+1) p+c} g(x)|u|^{r-2} u v d s
\end{aligned}
$$

It is clear that Problem (1) has a variational structure. Let $I_{\lambda}: W \rightarrow R$ be the corresponding energy functional of problem (1) is defined by

$$
I_{\lambda}(u)=\frac{1}{p} M(u)-\frac{\lambda}{q} A(u)-\frac{1}{r} B(u),
$$

where

$$
M(u)=\int_{\Omega}\left(|\nabla u|^{p}|x|^{-a p}\right) d x, A(u)=\int_{\Omega}|x|^{-(a+1) p+c} f(x)|u|^{q} d x
$$

and

$$
B(u)=\int_{\partial \Omega}|x|^{-(a+1) p+c} g(x)|u|^{r} d s .
$$

It is well known the weak solutions of equation (1) are the critical points of the energy functional $I_{\lambda}$. Let $J$ be the energy functional associated with an elliptic problem on a Banach space $X$. If $J$ is bounded below and $J$ has a minimizer on $X$, then this minimizer is a critical point of $J$. So, it is a solution of the corresponding elliptic problem. However, the energy functional $I_{\lambda}$, is not bounded below on the whole space $W$, but is bounded on an appropriate subset, and a minimizer on this set (if it exists) gives rise to solution to (1). Consider the Nehari minimization problem for $\lambda \in \mathbb{R} \backslash\{0\}$,

$$
\alpha_{\lambda}=\inf \left\{I_{\lambda}(u): u \in \mathcal{N}_{\lambda}\right\},
$$

where $\mathcal{N}_{\lambda}=\left\{u \in W \backslash\{0\}:\left\langle I_{\lambda}^{\prime}(u), u\right\rangle=0\right\}$. It is easy to see that $u \in \mathcal{N}_{\lambda}$ if and only if

$$
M(u)-\lambda A(u)=B(u) .
$$


Note that $\mathcal{N}_{\lambda}$ contains every nonzero solution of problem (1).

Define

$$
\Phi_{\lambda}(u)=\left\langle I_{\lambda}^{\prime}(u), u\right\rangle
$$

Then for $u \in N_{\lambda}$,

$$
\begin{aligned}
\left\langle\Phi_{\lambda}^{\prime}(u), u\right\rangle & =p M(u)-\lambda q A(u)-r B(u) \\
& =\lambda(p-q) A(u)-(r-p) B(u) \\
& =(p-q) M(u)-(r-q) B(u) \\
& =(p-r) M(u)-\lambda(q-r) A(u) .
\end{aligned}
$$

Now, we split $\mathcal{N}_{\lambda}$ into three parts:

$$
\begin{aligned}
& \mathcal{N}_{\lambda}^{+}=\left\{u \in N_{\lambda}:\left\langle\Phi_{\lambda}^{\prime}(u), u\right\rangle>0\right\}, \\
& \mathcal{N}_{\lambda}^{0}=\left\{u \in N_{\lambda}:\left\langle\Phi_{\lambda}^{\prime}(u), u\right\rangle=0\right\}, \\
& \mathcal{N}_{\lambda}^{-}=\left\{u \in N_{\lambda}:\left\langle\Phi_{\lambda}^{\prime}(u), u\right\rangle<0\right\} .
\end{aligned}
$$

To state our main result, we now present some important properties of $\mathcal{N}_{\lambda}^{+}, \mathcal{N}_{\lambda}^{0}$, and $\mathcal{N}_{\lambda}^{-}$.

Lemma 2.2. There exists $\lambda_{0}>0$ such that for $0<\lambda<\lambda_{0}$, we have $\mathcal{N}_{\lambda}^{0}=\emptyset$.

Proof. Suppose otherwise, thus for $\lambda_{0}=\left[\frac{(p-q)}{(r-q)(\bar{C})^{r}}\right]^{\frac{p-q}{r-p}}\left[\frac{(r-p)}{(r-q)} \frac{1}{C^{q}}\right]$, there exists $\lambda \in\left(0, \lambda_{0}\right)$ such that $\mathcal{N}_{\lambda}^{0} \neq \emptyset$. Then for $u \in \mathcal{N}_{\lambda}^{0}$ we have

$$
\begin{aligned}
0 & =\left\langle\Phi_{\lambda}^{\prime}(u), u\right\rangle=(p-r) M(u)+\lambda(r-q) A(u) \\
& =(p-q) M(u)+(q-r) B(u) .
\end{aligned}
$$

By the Sobolev imbedding theorem,

$$
A(u) \leq\|u\|_{q}^{q} \leq C^{q}\|u\|_{W}^{q}
$$

and

$$
B(u) \leq\|u\|_{r}^{r} \leq \bar{C}^{r}\|u\|_{W}^{r}
$$

By using $(9)-(10)$ in $(7)-(8)$ we get

$$
\|u\|_{W} \leq\left(\frac{\lambda(r-q)}{r-p} C^{q}\right)^{\frac{1}{p-q}}
$$


and

$$
\|u\|_{W} \geq\left(\frac{(p-q)}{(r-q) \bar{C}^{r}}\right)^{\frac{1}{r-p}} .
$$

This imoplies $\lambda \geq \lambda_{0}$, which is a contradiction. Thus, we can conclude that there exists $\lambda_{0}>$ 0 such that for $0<\lambda<\lambda_{0}$, we have $\mathcal{N}_{\lambda}^{0}=\emptyset$.

By (4) and (6), It is easy to see that the following lemma holds. We omit the straight forward details.

Lemma 2.3. We have

(i) If $u \in \mathcal{N}_{\lambda}^{+}$, then $A(u)>0$;

(ii) If $u \in \mathcal{N}_{\lambda}^{-}$, then $B(u)>0$.

By Lemma 2.2, for $0<\lambda<\lambda_{0}$ we write $\mathcal{N}_{\lambda}=\mathcal{N}_{\lambda}^{+} \cup \mathcal{N}_{\lambda}^{-}$and define

$$
\alpha_{\lambda}^{+}=\inf _{u \in \mathcal{N}_{\lambda}^{+}} I_{\lambda}(u) ; \alpha_{\lambda}^{-}(\Omega)=\inf _{u \in \mathcal{N}_{\lambda}^{-}} I_{\lambda}(u)
$$

Also, as proved in Binding, Drabek and Huang [8] or in Brown and Zhang [12], we have the following lemma.

Lemma 2.4. Suppose that $u_{0}$ is a local minimizer for $I_{\lambda}$ on $\mathcal{N}_{\lambda}$. Then, if $u_{0} \notin \mathcal{N}_{\lambda}^{0}, u_{0}$ is a critical point of $I_{\lambda}$.

Then we have the following result.

Lemma 2.5. $I_{\lambda}$ is coercive and bounded below on $N_{\lambda}$.

Proof. If $u \in \mathcal{N}_{\lambda}$, it follows from (2) and the Sobolev embedding theorem

$$
\begin{aligned}
I_{\lambda}(u) & =\left(\frac{r-p}{p r}\right) M(u)-\lambda\left(\frac{r-q}{q r}\right) A(u) \\
& \geq\left(\frac{r-p}{p r}\right) M(u)-\lambda\left(\frac{r-q}{q r}\right)\|u\|_{q}^{q}
\end{aligned}
$$




$$
\begin{aligned}
& \geq\left(\frac{r-p}{p r}\right) M(u)-\lambda\left(\frac{r-q}{q r}\right) C^{q}\|u\|_{W}^{q} . \\
& =\left(\frac{r-p}{p r}\right)\|u\|_{W}^{p}-\lambda\left(\frac{r-q}{q r}\right) C^{q}\|u\|_{W}^{q} .
\end{aligned}
$$

Thus $I_{\lambda}$ is coercive and bounded below on $N_{\lambda}$.

Lemma 2.6. Let $\lambda^{*}=\frac{(r-p)}{p q(r-q) C^{r}}\left[\frac{p-q}{(r-q) \bar{C}^{r}}\right]^{\frac{p-q}{r-p}}$. Then if $0<\lambda<\lambda^{*}$, We have

(i) $\alpha_{\lambda}^{+}<0$;

(ii) $\alpha_{\lambda}^{-} \geq k_{0}$, for some $k_{0}=k_{0}(q, r, C, \bar{C})$.

Proof. (i) Let $u \in \mathcal{N}_{\lambda}^{+}$. By (5)

$$
\frac{p-q}{r-q} M(u)>B(u)
$$

and so

$$
\begin{aligned}
I_{\lambda}(u) & =\left(\frac{1}{p}-\frac{1}{q}\right) M(u)+\left(\frac{1}{q}-\frac{1}{r}\right) B(u) \\
& \leq\left(\frac{q-p}{p q}\right) M(u)+\left(\frac{r-q}{q r}\right)\left[\frac{(p-q)}{(r-q)} M(u)\right] \\
& =\left[\frac{q-p}{p q}+\frac{p-q}{q r}\right] M(u) \\
& =\frac{(p-q)(p-r)}{p q r} M(u)<0 .
\end{aligned}
$$

Thus $\alpha_{\lambda}^{+}<0$.

(ii) Let $u \in \mathcal{N}_{\lambda}^{-}$, by (5) and the Sobolev trace imbedding theorem we have

$$
\begin{aligned}
M(u) & <\frac{r-q}{p-q} B(u) \leq \frac{r-q}{p-q}\|u\|_{r}^{r} \\
& \leq \frac{r-q}{p-q} \bar{C}^{r}\|u\|_{W}^{r} .
\end{aligned}
$$

This implies

$$
\|u\|_{W}>\left(\frac{p-q}{(r-q) \bar{C}^{r}}\right)^{\frac{1}{r-p}} \text { for all } u \in \mathcal{N}_{\lambda}^{-} .
$$


By (3) we have

$$
\begin{aligned}
I_{\lambda}(u) & \geq\|u\|_{W}^{q}\left[\frac{r-p}{p r}\|u\|_{W}^{p-q}-\lambda C^{q}\left(\frac{r-q}{q r}\right)\right] \\
& >\left(\frac{p-q}{(r-q) \bar{C}^{r}}\right)^{\frac{q}{r-p}}\left[\frac{r-p}{p r}\left(\frac{p-q}{(r-q) \bar{C}^{r}}\right)^{\frac{p-q}{r-p}}-\lambda C^{q}\left(\frac{r-q}{q r}\right] .\right.
\end{aligned}
$$

Thus, if

$$
0<\lambda<\lambda^{*}
$$

then

$$
I_{\lambda}>k_{0}, \text { for all } u \in \mathcal{N}_{\lambda}^{-}
$$

for some $k_{0}=k_{0}(p, q, r, C, \bar{C}, \lambda)>0$. This completes the proof.

For each $u \in W$ with $B(u)>0$, we write

$$
t_{\max }=\left(\frac{(p-q) M(u)}{(r-q) B(u)}\right)^{1 /(r-p)}>0 .
$$

Then we have the following lemma.

Lemma 2.7 For each $u \in W$ with $B(u)>0$ and $0<\lambda<\lambda_{0}=\left[\frac{(p-q)}{(r-q)(\bar{C})^{r}}\right]^{\frac{p-q}{r-p}}\left[\frac{(r-p)}{(r-q)} \frac{1}{C^{q}}\right]$, we have

(i) if $A(u) \leq 0$, then there is unique $t^{-}>t_{\max }$ such that $t^{-} u \in \mathcal{N}_{\lambda}^{-}$and

$$
I_{\lambda}\left(t^{-} u\right)=\sup _{t \geq 0} I_{\lambda}(t u)
$$

(ii) if $A(u)>0$, then there are unique $0<t^{+}=t^{+}(u)<t_{\max }<t^{-}$such that $t^{+} u \in \mathcal{N}_{\lambda}^{+}$, $t^{-} u \in \mathcal{N}_{\lambda}^{-}$and

$$
I_{\lambda}\left(t^{+} u\right)=\inf _{0 \leq t \leq t_{\max }} I_{\lambda}(t u), I_{\lambda}\left(t^{-} u\right)=\sup _{t \geq 0} I_{\lambda}(t u)
$$

Proof. Fix $u \in W$ with $B(u)>0$. Let

$$
E(t)=t^{p-q} M(u)-t^{r-q} B(u) \text { for } t \geq 0 .
$$

Clearly, $E(0)=0, E(t) \rightarrow-\infty$ as $t \rightarrow \infty$. Since

$$
E^{\prime}(t)=(p-q) t^{p-q-1} M(u)-(r-q) t^{r-q-1} B(u),
$$


we have $E^{\prime}(t)=0$ at $t=t_{\max }, E^{\prime}(t)>0$ for $t \in\left[0, t_{\max }\right)$ and $E^{\prime}(t)<0$ for $t \in\left(t_{\max }, \infty\right)$. Then $E(t)$ achieves its maximum at $t_{\max }$, increasing for $t \in\left[0, t_{\max }\right)$ and decreasing for $t \in\left(t_{\max }, \infty\right)$. Moreover,

$$
\begin{aligned}
E\left(t_{\max }\right) & =\left(\frac{(p-q) M(u)}{(r-q) B(u)}\right)^{\frac{p-q}{r-p}} M(u)-\left(\frac{(p-q) M(u)}{(r-q) B(u)}\right)^{\frac{r-q}{r-p}} B(u) \\
& =\|u\|_{W}^{q}\left[\left(\frac{p-q}{r-q}\right)^{\frac{p-q}{q-p}}-\left(\frac{p-q}{r-q}\right)^{\frac{r-q}{r-p}}\right]\left(\frac{\|u\|_{W}^{r}}{B(u)}\right)^{\frac{p-q}{r-p}} \\
& \geq\|u\|_{W}^{q}\left(\frac{r-p}{r-q}\right)\left(\frac{p-q}{r-q}(\bar{C})^{-r}\right)^{\frac{p-q}{r-p}} .
\end{aligned}
$$

(i) $A(u) \leq 0$ : There is a unique $t^{-}>t_{\max }$ such that $E\left(t^{-}\right)=\lambda A(u)$ and $E^{\prime}\left(t^{-}\right)<0$. Now,

$$
\begin{aligned}
& (p-q) M\left(t^{-} u\right)-(r-q) B\left(t^{-} u\right) \\
& =\left(t^{-}\right)^{1+q}\left[(p-q)\left(t^{-}\right)^{p-1-q} M(u)-(r-q)\left(t^{-}\right)^{r-q-1} B(u)\right] \\
& =\left(t^{-}\right)^{1+q} E^{\prime}\left(t^{-}\right)<0
\end{aligned}
$$

and

$$
\begin{aligned}
& \left\langle I_{\lambda}^{\prime}\left(t^{-} u\right), t^{-} u\right\rangle \\
& =\left(t^{-}\right)^{p} M(u)-\left(t^{-}\right)^{q} \lambda A(u)-\left(t^{-}\right)^{r} B(u) \\
& =\left(t^{-}\right)^{q}\left[\left(t^{-}\right)^{p-q} M(u)-\lambda A(u)-\left(t^{-}\right)^{r-q} B(u)\right] \\
& =\left(t^{-}\right)^{q}\left[E\left(t^{-}\right)-\lambda A(u)\right]=0 .
\end{aligned}
$$

Thus, $t^{-} u \in \mathcal{N}_{\lambda}^{-}$. Since for $t>t_{\max }$, we have

$$
\begin{gathered}
(p-q) M(t u)-(r-q) B(t u)<0, \\
\frac{d^{2}}{d t^{2}} I_{\lambda}(t u)<0
\end{gathered}
$$

and

$$
\frac{d}{d t} I_{\lambda}(t u)=t^{p-1} M(u)-\lambda t^{q-1} A(u)-t^{r-1} B(u)=0 \quad \text { for } t=t^{-} .
$$

Thus, $I_{\lambda}\left(t^{-} u\right)=\sup _{t \geq 0} I_{\lambda}(t u)$. 
(ii) $A(u)>0$. By (13) and

$$
\begin{aligned}
E(0) & =0<\lambda A(u) \\
& \leq \lambda C^{q}\|u\|_{W}^{q} \\
& <\|u\|_{W}^{q}\left(\frac{r-p}{r-q}\right)\left(\frac{p-q}{r-q}(\bar{C})^{-r}\right)^{\frac{p-q}{r-p}} \\
& \leq E\left(t_{\max }\right)
\end{aligned}
$$

for $0<\lambda<\lambda_{0}(p, q, r, C, \bar{C})$, there are unique $t^{+}$and $t^{-}$such that $0<t^{+}<t_{\max }<t^{-}$,

$$
\begin{gathered}
E\left(t^{+}\right)=\lambda A(u)=E\left(t^{-}\right), \\
E^{\prime}\left(t^{+}\right)>0>E^{\prime}\left(t^{-}\right) .
\end{gathered}
$$

We have $t^{+} u \in \mathcal{N}_{\lambda}^{+}, t^{-} u \in \mathcal{N}_{\lambda}^{-}$, and $I_{\lambda}\left(t^{-} u\right) \geq I_{\lambda}(t u) \geq I_{\lambda}\left(t^{+} u\right)$ for each $t \in\left[t^{+}, t^{-}\right]$and $I_{\lambda}\left(t^{+} u\right) \leq I_{\lambda}(t u)$ for each $t \in\left[0, t^{+}\right]$. Thus,

$$
I_{\lambda}\left(t^{+} u\right)=\inf _{0 \leq t \leq t_{\max }} I_{\lambda}(t u), I_{\lambda}\left(t^{-} u\right)=\sup _{t \geq 0} I_{\lambda}(t u)
$$

This completes the proof.

For each $u \in W$ with $\lambda A(u)>0$, we write

$$
\bar{t}_{\max }=\left(\frac{(r-q) \lambda A(u)}{(r-p) M(u)}\right)^{1 /(p-q)}>0 .
$$

Then we have the following lemma.

Lemma 2.8 For each $u \in W$ with $\lambda A(u)>0$, we have

(i) if $B(u) \leq 0$, then there is unique $t^{+}<\bar{t}_{\text {max }}$ such that $t^{+} u \in \mathcal{N}_{\lambda}^{+}$and

$$
I_{\lambda}\left(t^{+} u\right)=\inf _{t \geq 0} I_{\lambda}(t u)
$$

(ii) if $B(u)>0$, then there are unique $0<t^{+}=t^{+}(u)<\bar{t}_{\max }<t^{-}$such that $t^{+} u \in \mathcal{N}_{\lambda}^{+}$, $t^{-} u \in \mathcal{N}_{\lambda}^{-}$and

$$
I_{\lambda}\left(t^{+} u\right)=\inf _{0 \leq t \leq \bar{t}_{\max }} I_{\lambda}(t u), I_{\lambda}\left(t^{-} u\right)=\sup _{t \geq 0} I_{\lambda}(t u) .
$$

Proof. Fix $u \in W$ with $\lambda A(u)>0$. Let

$$
\bar{E}(t)=t^{p-r} M(u)-\lambda t^{q-r} A(u) \text { for } t>0 .
$$


Clearly, $\bar{E}(t) \rightarrow-\infty$ as $t \rightarrow 0^{+}$. Since

$$
\bar{E}^{\prime}(t)=(p-r) t^{p-r-1} M(u)-\lambda(q-r) t^{q-r-1} A(u),
$$

we have $\bar{E}^{\prime}(t)=0$ at $t=\bar{t}_{\max }, \bar{E}^{\prime}(t)>0$ for $t \in\left[0, \bar{t}_{\max }\right)$ and $\bar{E}^{\prime}(t)<0$ for $t \in\left(\bar{t}_{\max }, \infty\right)$. Then $\bar{E}(t)$ achieves its maximum at $\bar{t}_{\text {max }}$, increasing for $t \in\left[0, \bar{t}_{\max }\right)$ and decreasing for $t \in\left(\bar{t}_{\max }, \infty\right)$. Similar to argument in Lemma 2.7, we can obtain the result of Lemma 2.8. $\square$

\section{Existence result}

Now we can state our main result.

Theorem 3.1 If the parameter $\lambda$ satisfy $0<\lambda<\lambda^{*}$, then problem (1) has at least two solutions $u_{0}^{+}$and $u_{0}^{-}$such that $u_{0}^{ \pm} \geq 0$ in $\Omega$ and $u_{0}^{ \pm} \neq 0$.

The proof of this Theorem will be a consequence of the next two propositions.

Proposition 3.2 If $0<\lambda<\lambda^{*}$, then the functional $I_{\lambda}$ has a minimizer $u_{0}^{+}$in $\mathcal{N}_{\lambda}^{+}$and it satisfies

(i) $I_{\lambda}\left(u_{0}^{+}\right)=\alpha_{\lambda}^{+}$;

(ii) $u_{0}^{+}$is a nontrivial nonnegative solution of problem (1), such that $u_{0}^{+} \geq 0$ in $\Omega$ and $u_{0}^{+} \neq 0$.

Proof. Let $\left\{u_{n}\right\}$ be a minimizing sequence for $I_{\lambda}$ on $\mathcal{N}_{\lambda}^{+}$, i.e., $\lim _{n \rightarrow \infty} I_{\lambda}\left(u_{n}\right)=\inf { }_{u \in \mathcal{N}_{\lambda}^{+}} I_{\lambda}(u)$. Then by Lemma 2.5 and the Rellich-Kondrachov theorem, there exist a subsequence $\left\{u_{n}\right\}$ and $u_{0}^{+} \in W$ such that $u_{0}^{+}$is a solution of problem (1) and

$$
\begin{gathered}
u_{n} \rightarrow u_{0}^{+} \quad \text { weakly in } W, \\
u_{n} \rightarrow u_{0}^{+} \quad \text { strongly in } L^{r}(\partial \Omega) \text { and in } L^{q}(\Omega) .
\end{gathered}
$$

This implies

$$
\begin{gathered}
\lambda A\left(u_{n}\right) \rightarrow \lambda A\left(u_{0}^{+}\right) \quad \text { as } n \rightarrow \infty \\
B\left(u_{n}\right) \rightarrow B\left(u_{0}^{+}\right) \quad \text { as } n \rightarrow \infty .
\end{gathered}
$$

Since

$$
I_{\lambda}\left(u_{n}\right)=\left(\frac{r-p}{p r}\right) M\left(u_{n}\right)-\lambda\left(\frac{r-q}{q r}\right) A\left(u_{n}\right),
$$


and by Theorem $2.6(i)$

$$
I_{\lambda}\left(u_{n}\right) \rightarrow \alpha_{\lambda}^{+}<0 \text { as } n \rightarrow \infty .
$$

Letting $n \rightarrow \infty$, we see that $A\left(u_{0}\right)>0$. In particular $u_{0}^{+} \neq 0$. Now we prove that $u_{n} \rightarrow u_{0}^{+}$ strongly in $W$. Suppose otherwise, then

$$
\left\|u_{0}^{+}\right\|_{W}<\liminf _{n \rightarrow \infty}\left\|u_{n}\right\|_{W}
$$

Fix $u \in W$ with $A(u)>0$. Let

$$
K_{u}(t)=\bar{E}(t)-B(u)
$$

where $\bar{E}(t)$ is as in (15.) Clearly, $K_{u}(t) \rightarrow-\infty$ as $t \rightarrow 0^{+}$, and

$$
K_{u}(t) \rightarrow-B(u) \quad \text { as } t \rightarrow \infty
$$

Since $K_{u}^{\prime}(t)=\bar{E}^{\prime}(t)$, similar argument as in the proof of Lemma 2.8, we have $K_{u}(t)$ achieves its maximum at $\bar{t}_{\text {max }}$, is increasing for $t \in\left(0, \bar{t}_{\max }\right)$ and decreasing for $t \in\left(\bar{t}_{\max }, \infty\right)$, where

$$
\bar{t}_{\max }=\left(\frac{(r-q) \lambda A(u)}{(r-p) M(u)}\right)^{1 /(p-q)}>0,
$$

is as in (14). Since $\lambda A\left(u_{0}^{+}\right)>0$, by Lemma 2.8, there is unique $t_{0}^{+}<\bar{t}_{\max }$ such that $t_{0}^{+} u_{0}^{+} \in$ $\mathcal{N}_{\lambda}^{+}$and

$$
I_{\lambda}\left(t_{0}^{+} u_{0}^{+}\right)=\inf _{0 \leq t \leq \bar{t}_{\max }\left(u_{0}\right)} I_{\lambda}\left(t u_{0}^{+}\right) .
$$

Then

$$
\begin{aligned}
K_{u_{0}^{+}}\left(t_{0}^{+}\right) & =\left(t_{0}^{+}\right)^{p-r} M\left(u_{0}^{+}\right)-\lambda\left(t_{0}^{+}\right)^{q-r} A\left(u_{0}^{+}\right)-B\left(u_{0}^{+}\right) \\
& =\left(t_{0}^{+}\right)^{-r}\left(M\left(t_{0}^{+} u_{0}^{+}\right)-\lambda A\left(t_{0}^{+} u_{0}^{+}\right)-B\left(t_{0}^{+} u_{0}^{+}\right)\right) \\
& =0 .
\end{aligned}
$$

By (16) and (17) we obtain

$$
K_{u_{n}}\left(t_{0}^{+}\right)>0 \quad \text { for } \mathrm{n} \text { sufficiently large. }
$$

Since $u_{n} \in \mathcal{N}_{\lambda}^{+}$, we have $\bar{t}_{\text {max }}\left(u_{n}\right)>1$. Moreover,

$$
K_{u_{n}}(1)=M\left(u_{n}\right)-\lambda A\left(u_{n}\right)-B\left(u_{n}\right)=0,
$$


and $K_{u_{n}}(t)$ is increasing for $t \in\left(0, \bar{t}_{\max }\left(u_{n}\right)\right)$. This implies $K_{u_{n}}(t)<0$ for all $t \in(0,1]$ and $\mathrm{n}$ sufficiently large. We obtain $1<t_{0}^{+} \leq \bar{t}_{\max }\left(u_{0}\right)$. But $t_{0}^{+} u_{0}^{+} \in \mathcal{N}_{\lambda}^{+}$and

$$
I_{\lambda}\left(t_{0}^{+} u_{0}^{+}\right)=\inf _{0 \leq t \leq \bar{t}_{\max }\left(u_{0}\right)} I_{\lambda}\left(t u_{0}^{+}\right) .
$$

This implies

$$
I_{\lambda}\left(t_{0}^{+} u_{0}^{+}\right)<I_{\lambda}\left(u_{0}^{+}\right)<\lim _{n \rightarrow \infty} I_{\lambda}\left(u_{n}\right)=\alpha_{\lambda}^{+},
$$

which is a contradiction. Hence

$$
u_{n} \rightarrow u_{0}^{+} \quad \text { strongly in } W
$$

This implies

$$
I_{\lambda}\left(u_{n}\right) \rightarrow I_{\lambda}\left(u_{0}^{+}\right)=\alpha_{\lambda}^{+} \quad \text { as } n \rightarrow \infty .
$$

Thus $u_{0}^{+}$is a minimizer for $I_{\lambda}$ on $\mathcal{N}_{\lambda}^{+}$. Since $I_{\lambda}\left(u_{0}^{+}\right)=I_{\lambda}\left(\left|u_{0}^{+}\right|\right)$and $\left|u_{0}^{+}\right| \in \mathcal{N}_{\lambda}^{+}$, by Lemma 2.4 we may assume that $u_{0}^{+}$is a nontrivial nonnegative solution of equation (1).

Next, we establish the existence of a local minimum for $I_{\lambda}$ on $\mathcal{N}_{\lambda}^{-}$.

Proposition 3.3 If $0<\lambda<\lambda^{*}$, then the functional $I_{\lambda}$ has a minimizer $u_{0}^{-}$in $\mathcal{N}_{\lambda}^{-}$and it satisfies

(i) $I_{\lambda}\left(u_{0}^{-}\right)=\alpha_{\lambda}^{-}$;

(ii) $u_{0}^{-}$is a nontrivial nonnegative solution of problem (1), such that $u_{0}^{-} \geq 0$ in $\Omega$ and $u_{0}^{-} \neq 0$.

Proof. Let $\left\{u_{n}\right\}$ be a minimizing sequence for $I_{\lambda}$ on $\mathcal{N}_{\lambda}^{-}$, i.e., $\lim _{n \rightarrow \infty} I_{\lambda}\left(u_{n}\right)=\inf { }_{u \in \mathcal{N}_{\lambda}^{-}} I_{\lambda}(u)$. Then by Lemma 2.25 and the Rellich-Kondrachov theorem, there exist a subsequence $\left\{u_{n}\right\}$ and $u_{0}^{-} \in W$ such that $u_{0}^{-}$is a solution of problem (1) and

$$
\begin{gathered}
u_{n} \rightarrow u_{0}^{-} \quad \text { weakly in } W, \\
u_{n} \rightarrow u_{0}^{-} \quad \text { strongly in } L^{r}(\partial \Omega) \text { and in } L^{q}(\Omega) .
\end{gathered}
$$

This implies

$$
\begin{aligned}
& A\left(u_{n}\right) \rightarrow A\left(u_{0}^{-}\right) \quad \text { as } n \rightarrow \infty \\
& B\left(u_{n}\right) \rightarrow B\left(u_{0}^{-}\right) \quad \text { as } n \rightarrow \infty .
\end{aligned}
$$

Moreover, by (5) we obtain

$$
B\left(u_{n}\right)>\frac{p-q}{r-q} M\left(u_{n}\right)
$$


By (12) and (18) there exists a positive number $\eta_{0}$ such that

$$
B\left(u_{n}\right)>\eta_{0}
$$

This implies

$$
B\left(u_{0}^{-}\right) \geq \eta_{0}
$$

Now we prove that $u_{n} \rightarrow u_{0}^{-}$strongly in $W$. Suppose otherwise, then

$$
\left\|u_{0}^{-}\right\|_{W}<\liminf _{n \rightarrow \infty}\left\|u_{n}\right\|_{W}
$$

By Lemma 2.7, there is unique $t_{0}^{-}$such that $t_{0}^{-} u_{0}^{-} \in \mathcal{N}_{\lambda}^{-}$. Since $\left\{u_{n}\right\} \in \mathcal{N}_{\lambda}^{-}, I_{\lambda}\left(u_{n}\right) \geq I_{\lambda}\left(t u_{n}\right)$ for all $t \geq 0$, we have

$$
I_{\lambda}\left(t_{0}^{-} u_{0}^{-}\right)<\lim _{n \rightarrow \infty} I_{\lambda}\left(t_{0}^{-} u_{n}\right) \leq \lim _{n \rightarrow \infty} I_{\lambda}\left(u_{n}\right)=\alpha_{\lambda}^{-},
$$

and this is a contradiction. Hence

$$
u_{n} \rightarrow u_{0}^{-} \quad \text { strongly in } W \text {. }
$$

This implies

$$
I_{\lambda}\left(u_{n}\right) \rightarrow I_{\lambda}\left(u_{0}^{-}\right)=\alpha_{\lambda}^{-} \text {as } n \rightarrow \infty .
$$

Since $I_{\lambda}\left(u_{0}^{-}\right)=I_{\lambda}\left(\left|u_{0}^{-}\right|\right)$and $\left|u_{0}^{-}\right| \in \mathcal{N}_{\lambda}^{-}$, by Lemma 2.34 and (19) we may assume that $u_{0}^{-}$is a nontrivial nonnegative solution of equation (1).

Proof of Theorem 3.1. By Propositions 3.2, 3.3, we obtain equation (1) has two nontrivial nonnegative solutions $u_{0}^{+}$and $u_{0}^{-}$such that $u_{0}^{+} \in \mathcal{N}_{\lambda}^{+}$and $u_{0}^{-} \in \mathcal{N}_{\lambda}^{-}$. It remain to show that the solutions found in Propositions 3.2 and 3.3 are distinct. Since $\mathcal{N}_{\lambda}^{+} \cap \mathcal{N}_{\lambda}^{-}=\emptyset$, this implies that $u_{0}^{+}$and $u_{0}^{-}$are distinct. This concludes the proof.

\section{References}

[1] C.O. Alves and A. El Hamidi, Nehari manifold and existence of positive solutions tob a class of quasilinear problems, Nonl. Anal, 60 (2005), 611-624.

[2] A. Ambrosetti, H. Brezis and G. Cerami, Combined effects of concave and convex nonlinearities in some elliptic problems, J. Funct. Anal, 122 (1994) 519-543. 
[3] H. Amman, J. Lopez-Gomez, A priori bounds and multiple solution for superlinear indefinite elliptic problems, J. Differential Equations, 146 (1998), 336-374.

[4] D. Arcoya and J.I. Diaz, S-shaped bifurcation branch in a quasilinear multivalued model arising in climatology, J. Differential Equations, 150 (1998), 215-225.

[5] C. Atkinson and K. El Kalli. Some boundary value problems for the Bingham model, J. Non-Newtonian Fluid Mech, 41 (1992), 339-363.

[6] C. Atkinson and C.R. Champion, On some boundary value problems for the equation $\nabla(F(|\nabla w|) \nabla w)=0$, Proc. R. Soc. London A, 448 (1995), 269-279.

[7] P.A. Binding, P. Drabek, Y.X. Huang, Existence of multiple solutions of critical quasilinear elliptic Neuman problems, Electron. J. Differential Equations, 5 (1997) 1-11.

[8] P.A. Binding, P. Drabek, Y.X. Huang, On Neuman boundary value problems for some quasilinear equations, Nonl. Anal, 42 (2000), 613-629.

[9] K. J. Brown, The Nehari manifold for a semilinear elliptic equation involving a sublinear term, Calc. Var, 22 (2005), 483-494.

[10] K. J. Brown and T.F. Wu, A fibering map approach to a semilinear elliptic boundary value problem, Electron. J. Differential Equations, 69 (2007),1-9.

[11] K. J. Brown and T.F. Wu, A semilinear elliptic system involving nonlinear boundary condition and sign changing weight function, J. Math. Anal. Appl, 337 (2008) 1326-1336.

[12] K. J. Brown and Y. Zhang, The Nehari manifold for a semilinear elliptic problem with a sign changing weight function, J. Differential Equations, 193, (2003), 481-499.

[13] M. del Pino and C. Flores, Asymptotic behavior of best constants and extremals for trace embeddings in expanding domains, Comm. Partial Differential Equations, 26 (1112) (2001), 2189-2210.

[14] J.I. Diaz, Nonlinear partial differential equations and free boundaries, Pitman Publ. Program 1985.

[15] P. Drabek and S.I. Pohozaev, Positive solutions for the p-Laplacian: application of the fibering method, Proc. Royal Soc. Edinburgh Sect A, 127 (1997) 721-747. 
[16] J. F. Escobar, Uniqueness theorems on conformal deformations of metrics, Sobolev inequalities, and an eigenvalue estimate, Comm. Pure Appl. Math, 43 (1990), 857-883.

[17] J. Fernández Bonder and J.D. Rossi, Asymptotic behavior of the best Sobolev trace constant in expanding and contracting domains, Comm. Pure Appl. Anal, 1 (2002), no. $3,359-378$.

[18] J. Fernández Bonder, E. Lami-Dozo and J.D. Rossi, Symmetry properties for the extremals of the Sobolev trace embedding, Ann. Inst. H. Poincaré Anal. Non Linèaire, 21 (2004), no. 6, 795-805.

[19] J. Fernández Bonder, S. Martínez and J.D. Rossi, The behavior of the best Sobolev trace constant and extremals in thin domains, J. Differential Equations, 198 (2004), no. 1, 129-148.

[20] P. Tolksdorf, Regularity for a more general class of quasilinear elliptic equations, J. Differential Equations, 51 (1984), 126-150.

[21] T. F. Wu, On semilinear elliptic equations involving concave-convex nonlinearities and sign-changing weight function, J. Math. Anal. Appl, 318 (2006) 253-270.

[22] T. F. Wu, A semilinear elliptic problem involving nonlinear boundary condition and sign-changing potential, Electron. J. Differential Equations, 131 (2006) 1-15.

[23] T. F. Wu, Multiplicity of positive solution of p-Laplacian problems with sign-changing weight function, Int. J. Math. Anal, 1 (2007),no. 12, 557-563.

[24] T. F. Wu, Multiplicity results for a semilinear elliptic equation involving sign-changing weight function, Rocky Mountain J. Math, (in press). 